\title{
La tutoría durante el proceso de desarrollo del TFG y TFM: análisis del grado de utilidad y satisfacción del alumnado
}

\section{Tutoring during the elaboration of Degree's and Master's Theses: analysis of utility and satisfaction as perceived by students}

\author{
Nuria Rebollo Quintela ${ }^{1}$ \\ nuria.rebollo@udc.es \\ Eva María Espiñeira Bellón \\ eva.espineira@udc.es \\ Universidade da Coruña, España
}

\section{Resumen:}

Los cambios introducidos por la creación del Espacio Europeo de Educación Superior y las exigentes demandas de la globalización y de la sociedad del conocimiento hacen que la orientación se deba erigir como un pilar fundamental. El propósito del presente estudio se centra en conocer el grado de utilización y satisfacción de la tutoría universitaria, concretamente durante el proceso de elaboración del Trabajo de Fin de Grado (TFG) y Trabajo de Fin de Máster (TFM) por parte de una muestra de egresados de los grados y másteres que se imparten en la Facultad de Ciencias de la Educación de la Universidade da Coruña. Se realiza, para ello, un estudio de carácter descriptivo, mediante la técnica de encuestación, aplicando un instrumento elaborado ad hoc sobre el mencionado proceso. Los resultados nos han permitido conocer y describir los perfiles del estudiantado durante su periodo formativo,

\begin{abstract}
:
The changes introduced by the creation of the European Higher Education Area and the ambitious demands of globalization and knowledge society mean that guidance must be pursued as a fundamental aim. The purpose of the present study is to explore use and satisfaction rates regarding university tutoring, specifically during the elaboration of Master's and Degree's theses by a sample of Graduates of the degrees and masters that are given in the Faculty of Sciences of the Education of the Universidade da Coruña. For this purpose, a descriptive study is carried out, using the survey technique, applying an instrument developed ad hoc for the aforementioned process. The results have allowed us to know and describe the profiles of students during their formative period, to verify that their perception of the usefulness of tutoring is optimal and finally to determine that their level of satisfaction with such
\end{abstract}

1 Dirección para correspondencia (correspondence address):

Nuria Rebollo Quintela. Área de Métodos de Investigación y Diagnóstico en Educación. Facultad de Ciencias de la Educación. Universidade da Coruña. Campus de Elviña, s/n. 15071 A Coruña (España) 
La tutoría durante el proceso de desarrollo del TFG y TFM: análisis del grado de utilidad y satisfacción del alumnado

Nuria Rebollo Quintela y Eva María Espiñeira Bellón

comprobar que la percepción que tienen sobre la utilidad de la tutoría es óptima y finalmente determinar que su nivel de satisfacción con dicha tutoría es elevado. El análisis realizado sobre el estado de la cuestión junto con los resultados obtenidos ponen de manifiesto la necesidad de seguir avanzando en esta temática, ya que quedan aspectos ligados a los retos de la labor docente como la definición del perfil idóneo del profesorado tutor para afrontar esta tarea.

\section{Palabras clave:}

Grado de satisfacción; grado de utilidad; tutoría; trabajo de fin de grado; trabajo de fin de máster; educación superior. tutoring is high. The analysis carried out on the state of the question together with the results obtained show the need to continue advancing in this subject, since there are aspects linked to the challenges of the teaching profession still to be pursued such as the definition of the ideal tutoring profile

\section{Key words:}

Satisfaction rate; usefulness rate; tutoring; degree's theses; master's theses; higher education.

\section{Résumé :}

Les modifications introduites par la création de l'Espace Européen d'Enseignement Supérieur et les attentes exigeantes de la mondialisation et de la société font que l'orientation doit être érigée comme un pilier fondamental. Le but de cette étude se concentre sur la connaissance du degré d'utilisation et de satisfaction du tutorat universitaire, en particulier au cours du processus de préparation des Travaux de fin de Licence (TFG) et de fin d'Étude en Master (TFM) à partir d'un échantillon d'étudiants de licences et masters enseignés à la Faculté des Sciences de l'Éducation de l'Université de A Coruña (Espagne). Il est fait, à cet effet, une étude descriptive, via des sondages, en utilisant un instrument développé de manière ad hoc concernant le processus ci-dessus. Les résultats nous ont permis de connaître et de décrire les profils des étudiants au cours de leur période de formation, de vérifier que la perception de l'utilité du mentorat est optimale et, enfin, de déterminer que le niveau de satisfaction des élèves avec un tel tutorat est élevé. L'analyse sur l'état de la question ainsi que les résultats obtenus montrent la nécessité de poursuivre les travaux d'investigation dans ce domaine, dû au fait que certaines questions restent à résoudre, notamment la définition du profil idéal de l'enseignant tuteur.

Mots clés:

Satisfaction; degré d'utilité; tutorat ; le travail de fin de licence; le travail de fin de master; l'enseignement supérieur.

Fecha de recepción: 8-3-2017

Fecha de aceptación: 29-5-2017 


\section{Planteamiento del problema o tema objeto de estudio}

En la actualidad nos encontramos en un momento preciso, óptimo y necesario para llevar a cabo una evaluación de todos los aspectos relacionados con el proceso de desarrollo del TFG/TFM y en concreto con lo estrechamente relacionado con la tutorización del mismo. Ya que, tal y como señalaban Álvarez y Pascual, en 2012 había "imposibilidad de valorar, de manera mínimamente rigurosa, el grado de satisfacción" de los agentes implicados en el desarrollo de los trabajos, ni de "constatar de manera práctica las ventajas e inconvenientes de los protocolos" (p.100) diseñados para ello, debido a la corta andadura de los nuevos títulos.

Es por ello, que el curso 2015/2016 (en el que se desarrolla el presente estudio) es el momento propicio para la recogida de resultados, ya que en la Universidade da Coruña han pasado cuatro años desde la primera promoción de los Grados y siete de Másters, permitiéndonos la experiencia acumulada "estar en condiciones de abordar diferentes aspectos" (Martínez-Fuentes y Pastor Seller, 2014, p.83).

Los trabajos que ponen fin a un título (TFG/TFM) conllevan "una labor de tutoría muy importante que se entiende como un nuevo espacio educativo de reflexión donde el alumnado puede exponer sus percepciones, reflexiones, problemas y objetivos, y el tutor/a puede enseñarle a aprender a aprender" (Rekalde Rodríguez, 2011,p. 188), adoptando el profesorado tutor una "especial relevancia $[. . .$.$] ya que las orientaciones$ y la información que proporciona al alumno y la supervisión que hace de su trabajo se convierten en requisito ineludible" (Martínez, Martínez y Pérez, como se citó en Martínez-Fuentes y Pastor Seller (2014, p. 84).

Percibiendo la necesidad de ahondar en este tema, surge esta investigación que tiene como propósito conocer el grado de utilización y satisfacción de la tutoría universitaria, concretamente durante el proceso de elaboración del TFG y TFM, por parte de una muestra de egresados/as de diferentes Grados y Másteres que se imparten en la Facultad de Ciencias de la Educación de la Universidade da Coruña (UDC).

\section{Antecedentes y fundamentación teórica}

El presente artículo aborda la experiencia del diseño y desarrollo de los TFG y los TFM desde el punto de vista de las tutorías efectuadas por el 
La tutoría durante el proceso de desarrollo del TFG y TFM: análisis del grado de utilidad y satisfacción del alumnado

Nuria Rebollo Quintela y Eva María Espiñeira Bellón

alumnado durante todo el proceso, para lo que se tiene en cuenta la normativa existente, los avances teóricos efectuados y la trayectoria de este trabajo académico.

\section{El TFG y el TFM en el Espacio Europeo de Educación Superior}

La configuración del nuevo Espacio Europeo de Educación Superior (EEES), junto con los cambios organizativos y estructurales experimentados en el sistema universitario español, supuso la promulgación de la Ley Orgánica 6/2001, de 21 de diciembre, de universidades, y la Ley Orgánica 4/2007, de 12 de abril. Dichas leyes se concretan con la aprobación del Real Decreto 1393/2007, de 29 de octubre, por el que se establece la ordenación de las enseñanzas universitarias oficiales, modificado por el Real Decreto 861/2010, de 2 de julio que tienen por objeto desarrollar la estructura de las enseñanzas, adaptadas al EEES.

La finalidad que se persigue con las enseñanzas de grado y de máster, serán: "la obtención por parte del estudiante de una formación general, en una o varias disciplinas, orientada a la preparación para el ejercicio de actividades de carácter profesional" (RD 1393/2007, artículo 9.1) y "la adquisición por el estudiante de una formación avanzada, de carácter especializado o multidisciplinar, orientada a la especialización académica o profesional, o bien a promover la iniciación en tareas investigadoras" (RD 1393/20017, artículo 10.1) respectivamente, teniendo en cuenta que, en la memoria de verificación de los nuevos planes de estudios ha de desarrollarse el diseño curricular del título propuesto, en el que conste el proyecto final de grado o de máster (artículo 6.4), siendo como enuncia Caicedo Camacho (2015) un requisito necesario para la obtención del título universitario. Por tanto, la incorporación del TFG y el TFM en los títulos emana, en última instancia, del "gran cambio implantado por el Espacio Europeo de Educación Superior (EEES), más conocido como proceso Bolonia" (Caicedo Camacho, 2015, p. 2). Sin olvidar, que este cambio no es algo que atañe exclusivamente al nuevo alumnado, sino que además, "desde el primer año de implantación de los nuevos Grados, se ha venido ofertando a los egresados de las titulaciones antiguas, Diplomaturas, la posibilidad de adaptar su cualificación profesional al nuevo sistema de títulos" (Ortiz-Repiso, García Zorita, Pacios y Vianello, 2014, p. 281). Tampoco podemos pasar por alto que, 
en el ámbito nacional, la elaboración y defensa de un trabajo final de carrera para la obtención de los estudios, sólo supone cierta novedad, ya que esta práctica se venía realizando en titulaciones de carácter técnico como es el caso de las ingenierías.

Por último, si bien el Real Decreto 1393/2007, regula la necesidad de incorporación de un proyecto final, este no explica convenientemente, tal y como señalan Álvarez y Pascual (2012) y Caicedo Camacho (2015), en qué consistirá dicho trabajo salvo en lo que concierne al momento en que se realiza y al número de créditos de que puede constar. En este sentido, se establece que:

- El TFG tendrá un mínimo de 6 créditos y un máximo de 12.5 por ciento del total de los créditos del título, deberá desarrollarse en la fase final del plan de estudios y ha de estar orientado a la evaluación de competencias asociadas al título (artículo 12.7).

- Las enseñanzas de Máster "concluirán con la elaboración y defensa pública de un trabajo de fin de Máster, que tendrá entre 6 y 30 créditos" (artículo 15.3).

Es por ello que "muchas universidades han establecido su propia normativa de carácter general lo suficientemente flexible para que cada Facultad o centro lo adapte a su propia especificidad" (Rekalde Rodríguez, 2011, p. 181)

\section{El TFG y el TFM en la UDC y en los títulos de la Facultad de Ciencias de la Educación}

Conviene en estos momentos empezar a enunciar las características concretas de los títulos abordados en esta investigación centrándonos en cinco aspectos fundamentales: la normativa propia del trabajo de fin de grado o máster en la Universidade da Coruña y en la Facultad de Ciencias de la Educación de la UDC, la figura del profesorado tutor (el proceso de designación de profesorado tutor de TFG/TFM, la propuesta y elección de líneas temáticas o tutor/a y las funciones de éste).

En cuanto al marco normativo que regula el TFG/TFM en la Universidad de la Coruña cabe destacar, que anualmente, se publica, por resolución rectoral, la Normativa de Gestión Académica, en la que se indica que las enseñanzas oficiales de Grado y Máster concluirán con la elabo- 
ración y defensa pública de un TFG o de un TFM en los que deben integrarse y desarrollarse los contenidos formativos recibidos, así como las capacidades, competencias y habilidades adquiridas durante el período de docencia y que supondrá la realización, por parte de cada estudiante y de forma individual, de un proyecto, una memoria o un estudio concreto. Asimismo, se dispone de la Normativa reguladora de la matrícula y la defensa del TFG y del TFM (aprobada por Consejo de Gobierno a 19 de diciembre de 2013 y modificada por Consejo de Gobierno a 30 de abril y 24 de julio de 2014, a 29 de enero de 2015 y a 28 de junio de 2016), en la cual, además de lo anteriormente mencionado, desde el curso 2016/2017, se incluye la posibilidad de que el TFG se pueda realizar de manera individual o en grupo. En este último caso, el centro ha de tener prevista esta modalidad en su reglamento, fundamentalmente como proyecto coordinado.

Es por ello que, la definición administrativa de los TFG y de los TFM corresponde a cada uno de los centros universitarios, dependiendo de su complejidad organizativa y del número de estudiantes matriculados/ as en estas asignaturas. Así, algunos centros dejan "a merced de cada departamento el desarrollo de su propio marco de acción" (Roiss, 2015, p. 275), o a la del órgano de coordinación del título, que "delegue en los respectivos departamentos" (Garrote de Marcos, 2015, p. 7).

En el caso de la Facultad de Ciencias de la Educación de la UDC, el Decanato elaboró y aprobó, en Junta de Facultad, de 25 de noviembre de 2011 (con el informe favorable de la comisión de planes de estudio de la UDC en su reunión de 22 de junio de 2012), el Reglamento para la elaboración del TFG en la Facultad de Ciencias de la Educación, de tal forma que todos los grados de dicha facultad (Grado en Educación Infantil, Educación Primaria, Educación Social y Logopedia) asumieron unas directrices sobre el TFG, en el marco fijado por toda la normativa aludida, que son las que resultan operativas hoy en día, con las posibles particularidades que se recojan en las guías docentes, elaboradas por cada una de las Comisiones de Titulación, respetando lo establecido en este reglamento y aprobadas en Junta de Facultad.

Con respecto a los Másteres de la Facultad, es la Comisión Académica de cada uno de ellos, la que tiene atribuidas estas funciones, desarrollándose un documento marco por cada uno: Máster Universitario en Didácticas Específicas; Máster Universitario en Dirección, Gestión e Innovación de Instituciones Escolares y Socioeducativas; Máster Univer- 
sitario en Estudios Avanzados sobre la Comunicación, el Lenguaje y sus Patologías; Máster Universitario en Profesorado de Educación Secundaria Obligatoria y Bachillerato, Formación Profesional y Enseñanzas de Idiomas; Máster Universitario en Psicología Aplicada y Máster Universitario en Psicopedagogía.

Normalmente, los documentos marco de elaboración de los TFG/ TFM se recogen como un guion a seguir en los cuales se establecen cuestiones relacionadas con el número de créditos y horas, las modalidades de TFG/TFM que se pueden presentar, la asignación de la dirección o tutorización de dicho trabajo, las líneas temáticas que se ofertan, los criterios de evaluación,... convirtiéndose en "una guía clara de los procedimientos que deben seguir para superar con éxito su trabajo", aspecto manifiesto por el alumnado (Ortiz-Repiso, et al., 2014, p. 300)

\section{El profesorado tutor y su designación}

Este proceso de tutorización, como destaca Rekalde Rodríguez (2011) puede ser realizado "por más de un director/tutor, pero no más de dos. No obstante, son pocos los casos en los que este hecho se recoge de forma excepcional", (p.184). En concreto, en la Facultad de Ciencias de la Educación de la UDC, los TFG y TFM serán dirigidos por un miembro del profesorado de los departamentos con docencia en el plan de estudios del título de Grado o Máster, y, en el caso del Máster Universitario en Profesorado de Educación Secundaria Obligatoria y Bachillerato, Formación Profesional y Enseñanzas de Idiomas, la dirección del TFM estará a cargo del profesorado de las materias de la especialidad cursada. Así mismo, se establece como criterio a tener en cuenta para formar parte del grupo de profesorado tutor, la pertenencia a un determinado departamento/especialidad, en contraposición con otras universidades que resaltan las cualidades personales, sociales y académicas (López Martín, González Villanueva y Velasco Quintana, 2013) que habrá de poseer el profesorado tutor en relación a "sus habilidades, no sólo académicas sino también interpersonales" (Zumaquero Gil, 2015). En este sentido, Martínez Clares, Pérez Cusó y Martínez Juárez (2014), identifican diferentes competencias profesionales del profesorado tutor relacionadas, con los conocimientos del profesorado tutor (sobre tutoría y sobre la estructura, organización y proyección profesional de las titulaciones), con 
la aplicación de técnicas propias de la tutoría, características personales, relaciones intra e interpersonales y formulación y encajamiento de críticas. Con ello se dirime que el profesorado tutor/a no debe ser competente únicamente en una determinada materia sino que "también lo ha de ser en aspectos relacionados con la orientación y la formación integral de su alumnado a través de la tutoría" (Amor Almedina, 2016, p. 97).

\section{Propuesta y elección de líneas temáticas o tutor/a}

Los temas o líneas de los TFG/TFM se propondrán de forma razonada; no obstante, como señalan Hernández-Leo, et al. (2013), "el contenido de esta asignatura es diferente para cada uno de los alumnos, pudiendo ser propuesto por el director/tutor del trabajo, por el estudiante o consensuado entre ambos" (p. 259). En el caso de la Facultad de Ciencias de la Educación de la UDC, la propuesta será remitida a la dirección del centro o a la coordinación del máster desde los departamentos o áreas a los que estos les hicieran el encargo de los TFG/TFM. El alumnado podrá proponer a los departamentos temas o líneas para que sean incorporados en su oferta. Cada Comisión de Titulación de Grado o Comisión Académica de Máster aprobará y hará pública una lista con los temas así como con el número de estudiantes que pueden escoger cada tema y los criterios de asignación. Dicha lista garantizará un número de direcciones suficiente para la totalidad de los/las estudiantes matriculados/as en el TFG/TFM. Díez Bueso (2015), alude en relación a la normativa universitaria para asignación de tutores y tareas de tutores que "resulta imprescindible reflexionar sobre este particular y buscar lugares comunes que permitan avanzar en la concreción del papel del docente en la dirección" (p. 2).

En la Facultad de Ciencias de la Educación de la UDC, en el caso de los TFG, para dirimir las prioridades en la elección de tema o tutor/a, se valora como criterio el expediente académico. No obstante, este criterio de elección o, en último caso, el sorteo, "no tiene en cuenta las preferencias e inquietudes científicas del alumnado" (Roiss, 2015, p. 278), de tal forma que "éste no siempre queda satisfecho con la oferta" (Roiss, 2015, p. 279). Esta situación no se produce en los TFM del centro, donde el alumnado normalmente, con anterioridad al proceso de elección, visita al profesorado para que le explique con mayor precisión lo que podría abordar en su proyecto con respecto a la línea temática publicada. 


\section{Funciones del profesorado tutor de TFG/TFM (qué, cómo y cuándo)}

El Real Decreto 1393/2007, no se refiere "a las funciones que debe desarrollar el profesor-tutor, dejando autonomía a las Universidades para que se pronuncien sobre este particular" (Zumaquero Gil, 2015, p. 2), siendo los centros los encargados de regularlas (Merino Tejedor, Pinedo González y Gómez Barreto, 2014).

En el caso de la Facultad de Ciencias de la Educación de la UDC, el profesor/a tutor/a de TFG será responsable de exponerle al alumnado, mediante un proceso de tutorización, las características del trabajo y deberá orientarlo en su desarrollo; realizará un seguimiento de la elaboración del TFG; velará por el cumplimiento de los objetivos fijados; y, finalmente, autorizará la presentación y defensa del TFG. Se recogen, por tanto, las mismas finalidades que las establecidas por Rekalde Rodríguez (2011). Con respecto a los TFM, podemos ampliar otras funciones más relacionadas con el proceso de investigación: ayudar al alumnado a concretar el tema sobre el que se trabajará, asesorar al alumnado respecto a la estructura del TFM, métodos de investigación, fuentes documentales y redacción.

Díez Bueso (2015) señala que los momentos en los que se produce la tutorización son durante el proceso de elaboración, de su presentación oral y en su evaluación, quien indica que "pese a la existencia de este tipo de regulaciones, en la mayor parte de Universidades españolas, un rasgo relativamente habitual es la falta de precisión normativa sobre las responsabilidades del profesor en la dirección del Trabajo" (p. 2).

Si nos centramos ahora en el cómo se ha de producir la tutorización, podemos determinar diferentes métodos a seguir teniendo en cuenta tanto los modos de efectuar la tutoría como los instrumentos que podemos emplear para ello:

- Mediante "un programa/cronograma presencial a modo de Taller o Seminario que preparase metodológicamente en la elaboración del TFG" (Fernández Riveira, 2015, p. 12).

- Estableciendo una "tutorización con hitos preestablecidos" (Hernández-Leo, et al., 2013, p. 266).

- Proponiendo "un calendario de tutorías y marcando unos objetivos mínimos que el alumno deberá alcanzar en cada una de ellas" (Zumaquero Gil, 2015, p. 4). 
Con respecto a los tipos de tutorías que pueden determinarse, se señala la importancia de efectuar inicialmente una tutoría grupal en la que el profesorado tutor indique las implicaciones formales que demanda el desarrollo de un TFG/TFM (Merino Tejedor, et al., 2014; Zumaquero Gil, 2015), siendo esto una práctica habitual en la Facultad de Ciencias de la Educación de la UDC. No obstante, Rekalde Rodríguez (2011) señala que "en otros centros se hace hincapié en las tutorías individualizadas, donde un tutor específico, le proporciona el tema concreto de su trabajo, así como toda la información y recursos necesarios para comenzar su desarrollo" (p. 185).

\section{Marco empírico}

\section{Objetivos}

Considerando la tutoría universitaria como un factor de calidad de la Educación Superior y teniendo como finalidad última conocer y valorar la percepción que tienen los egresados sobre el proceso de tutoría de TFG/TFM, los objetivos que se persiguen con el presente estudio son:

- conocer y describir los perfiles del estudiantado durante su periodo formativo,

- analizar la percepción que tiene sobre la utilidad que ha tenido la tutoría y

- determinar su nivel de satisfacción con la tutoría recibida.

\section{Metodología}

En esta investigación se ha optado por una metodología cuantitativa no experimental de tipo descriptivo- exploratoria ya que nuestro propósito radica en describir y analizar una realidad dada, medible y concreta (Pulido, Ballén y Zúñiga, 2007) a través de la técnica de la encuesta.

\section{Participantes}

Para el presente trabajo, se ha tenido en cuenta el alumnado matriculado en el TFG/TFM, durante el curso 2015/2016, estableciendo los siguientes criterios de exclusión: 
- No se consideran aquellos títulos no relacionados directamente con el ámbito escolar: Grado en Educación Social y Máster Universitario en Psicología Aplicada.

- No se consideran aquellos títulos cuyo TFG/TFM no tenga una asignación de 6 créditos ECTS: Grado en Logopedia (10 créditos ECTS), Máster Universitario en Didácticas Específicas (9 créditos ECTS) y Máster Universitario en Estudios Avanzados sobre la Comunicación, el Lenguaje y sus Patologías (12 créditos ECTS).

- No se considera el Máster Universitario en Profesorado de Educación Secundaria Obligatoria y Bachillerato, Formación Profesional y Enseñanzas de Idiomas, por no tener accesibilidad de contacto con dicha muestra.

Por lo tanto, se consideran participantes del estudio los siguientes títulos: Grado en Educación Infantil; Grado en Educación Primaria; Máster Universitario en Dirección, Gestión e Innovación de Instituciones Escolares y Socioeducativas y Máster Universitario en Psicopedagogía.

\section{Muestra}

El universo-población de este estudio lo conformaban el alumnado del Grado de Educación Infantil (N=133), Educación Primaria (117), Máster Universitario en Psicopedagogía $(\mathrm{N}=33)$ y Máster Universitario en Dirección, Gestión e Innovación de Instituciones Escolares y Socioeducativas $(\mathrm{N}=17)$, que defendieron su trabajo de fin de grado o máster en el curso 2015/2016, siendo todos ellos títulos impartidos en la Facultad de Ciencias de la Educación de la Universidade da Coruña.

Se realizó un muestreo no probabilístico de tipo intencional para la selección de la muestra ya que "no es necesario obtener datos de todos los posibles sujetos para comprender con exactitud la naturaleza del fenómeno que se estudia, sino que, en general, se puede alcanzar esa comprensión con una parte de los sujetos" (Fox, 1981, p. 367).

Podemos decir que finalmente la muestra productora de datos está compuesta por 73 sujetos. 
La tutoría durante el proceso de desarrollo del TFG y TFM: análisis del grado de utilidad y satisfacción del alumnado

Nuria Rebollo Quintela y Eva María Espiñeira Bellón

\section{Instrumento}

Como instrumento de recogida de la información, se empleó el Cuestionario sobre el grado de utilidad y satisfacción con la tutoría del TFG/TFM ya que se pretende "conocer lo que hacen, opinan o piensan los encuestados mediante preguntas realizadas por escrito y que puedan ser respondidas sin la presencia del encuestador" (Buendía, Colás y Hernández, 1998, p. 124).

Para su diseño y elaboración se ha partido del marco teórico, de investigaciones recientes publicadas sobre el objeto de estudio (Cremades Andreu, García Gil, Ramírez Rico y Miraflores Gómez, 2016; López Martín, et al., 2013; Pérez Cusó, 2013; Tarí Guilló et al., 2015; Todd, Bannister y Clegg, 2004) así como de las principales directrices y pautas metodológicas propias de la construcción de este tipo de instrumento (Corbetta, 2007; Gil Pascual, 2011).

El cuestionario se estructura en dos grandes apartados:

- El primero en el que se recogen las preguntas de identificación que permiten describir la muestra objeto de estudio en relación con una serie de características personales y académicas.

- El segundo en el que se incluyen las preguntas básicas: 30 preguntas cerradas sobre el grado de utilidad y satisfacción con la tutoría y 2 preguntas abiertas en las que se ofrece la oportunidad de detectar puntos fuertes y propuestas de mejoras sobre el proceso de tutorización.

Para las preguntas cerradas se ha empleado una escala sumativa de tipo Likert ya que permite que el sujeto responda en base a su grado de acuerdo o desacuerdo con el ítem, indicando un juicio de valor del mismo (Gil Pascual, 2011). La escala es de 5 puntos, siendo 1 nada y 5 mucho.

El procedimiento seguido para su elaboración, así como su sometimiento a valoración de jueces expertos aseguran la validez de contenido del instrumento elaborado.

\section{Resultados y discusión}

Para el tratamiento de los datos, se utilizó el programa estadístico IBM Statistics SPSS 24. Los análisis realizados responden a medidas descriptivas de tendencia central y de dispersión. 
En cuanto a las características principales de la muestra, podemos decir que el $90.4 \%$ son mujeres y el $9.6 \%$ hombres, por lo que nos encontramos con una muestra feminizada, claro reflejo de la población objeto de estudio, ya que en los títulos del ámbito educativo la matrícula es principalmente femenina.

En cuanto a la titulación cursada en 2015/2016, la muestra se distribuye en Grado en Educación Infantil (50.7\%), Grado en Educación Primaria (32.9\%), Máster Universitario en Psicopedagogía (8.2\%) y Máster Universitario en Dirección, Gestión e Innovación de Instituciones Escolares y Socioeducativas (8.2\%).

El proceso de elección fue, bien por el interés hacia la temática independientemente de la persona que le tutorizara (54.8\% de los casos), por preferencia hacia el profesorado tutor $(32.9 \%)$ o el $12.3 \%$ no ha tenido la oportunidad de elegir (figura 1).

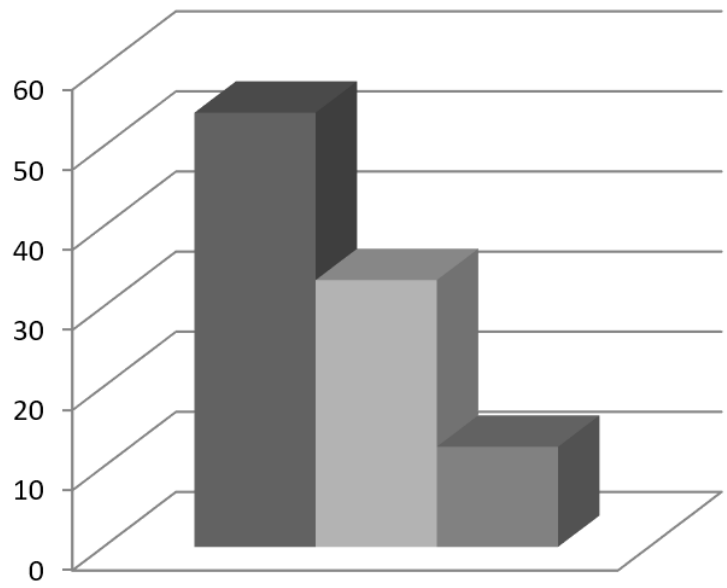

- He elegido la temática que me gustaba, independientemente de la persona que me tutorizase - He elegido el /la tutor/a que me gustaba

No he tenido la oportunidad de elegir

Figura 1. Proceso de elección del TFG/TFM.

\section{Características del profesorado tutor}

Partiendo de la consideración de la muestra respecto a la necesidad del trabajo realizado por el profesorado tutor universitario (95.9\%), los resultados manifiestan que el abanico de cualidades que el alumnado demanda en él se agrupan principalmente en tres dimensiones: características académicas, personales y sociales. Como se puede apreciar en la figura 2, si bien a todas ellas se le ha otorgado una gran relevancia, des- 
La tutoría durante el proceso de desarrollo del TFG y TFM: análisis del grado de utilidad y satisfacción del alumnado

Nuria Rebollo Quintela y Eva María Espiñeira Bellón

tacan en mayor medida las habilidades personales y sociales y en menor las relacionadas con los conocimientos de tipo académico, habiendo homogeneidad en las respuestas.

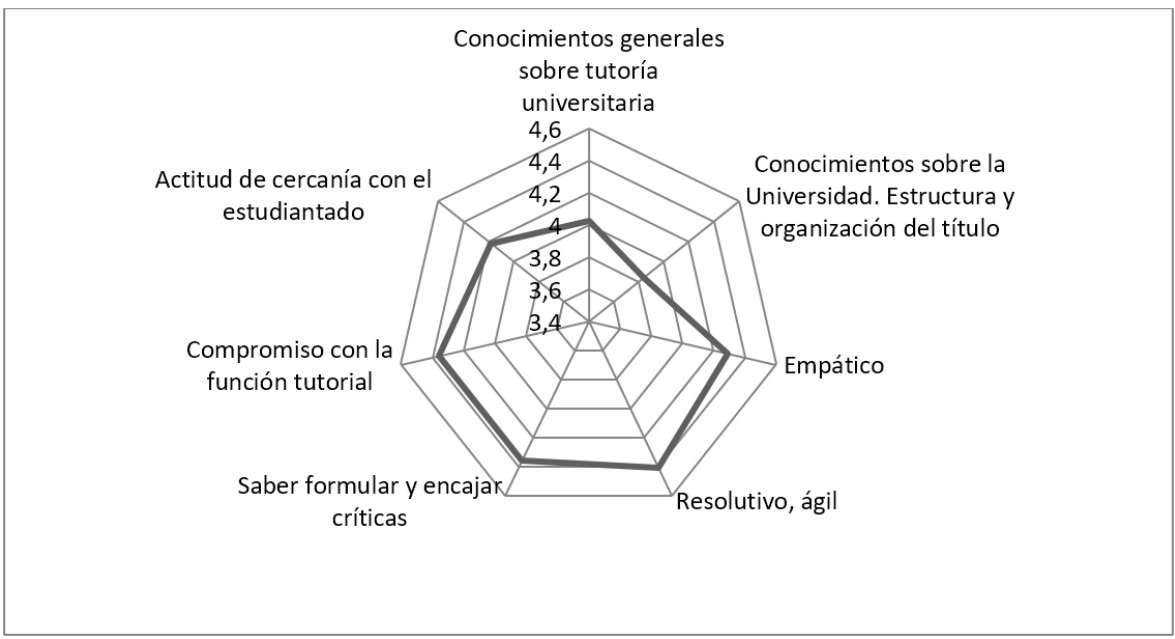

Figura 2. Características del profesorado tutor.

\section{Uso y utilidad de la tutoría}

La casi totalidad de la muestra encuestada indica que la tutoría es un aspecto necesario en su formación universitaria (94.5\%), usando en la mayor parte de los casos la de carácter individual $(\bar{x}=4.18)$ frente a la de carácter grupal $(\bar{x}=2.21)$. El uso que realizan de la misma aparece relacionado con la utilidad, en el caso de la tutoría individual $(\bar{x}=4.44)$, pero no en el caso de la grupal $(\bar{x}=3.37)$ (tabla 1$)$. En general, el alumnado refiere que el grado de utilidad de las tutorías ha sido bastante buena $(\bar{x}=4)$.

\section{Tabla 1. Frecuencia y utilidad de la tutoría.}

\begin{tabular}{|c|c|c|c|c|}
\hline & $\mathrm{N} \quad \bar{x}$ & Md & Mo & SD \\
\hline $\begin{array}{l}\text { ¿Con qué frecuencia has utilizado la tutoría indivi- } \\
\text { dual? }\end{array}$ & 734.18 & 5 & 5 & 1.059 \\
\hline ¿Con qué frecuencia has utilizado la tutoría grupal? & 732.21 & 2 & 1 & 1.213 \\
\hline ¿Qué utilidad ves a la tutoría individual? & 734.44 & 5 & 5 & .866 \\
\hline ¿Qué utilidad ves a la tutoría grupal? & 733.37 & 3 & 5 & 1.339 \\
\hline $\begin{array}{l}\text { En general, el grado de utilidad de las tutorías ha } \\
\text { sido... }\end{array}$ & 734.00 & 4 & 5 & .167 \\
\hline
\end{tabular}


El uso que se ha dado a esa tutoría ha radicado principalmente en aspectos relacionados con el seguimiento académico del trabajo y la resolución de dudas (con una media cercana al 4). En el extremo contrario nos encontramos con que el alumnado ha requerido en menor medida la colaboración del profesor tutor para el proceso de redacción y defensa del TFG/TFM (tabla 2).

Tabla 2. Usos dados a la tutoría.

\begin{tabular}{llllll}
\hline & $\mathrm{N}$ & $\bar{x}$ & $\mathrm{Md}$ & $\mathrm{Mo}$ & $\mathrm{SD}$ \\
\hline Proceso de redacción del TFG/TFM & 73 & 3.12 & 3 & 5 & 1.471 \\
Proceso de defensa del TFG/TFM & 73 & 2.79 & 3 & 3 & 1.354 \\
Información sobre el TFG/TFM & 73 & 3.34 & 3 & 3 & 1.261 \\
Seguimiento académico del TFG/TFM & 73 & 4.07 & 5 & 5 & 1.171 \\
Resolución de dudas del TFG/TFM & 73 & 4.03 & 4 & 5 & 1.166 \\
\hline
\end{tabular}

\section{Satisfacción respecto a la tutoría}

Por último, se ha preguntado al alumnado respecto a su satisfacción con la tutoría del TFG/TFM, en la línea de las cualidades demandas en el profesor/a tutor/a. Es decir, saber en qué medida el profesorado tutor que había tenido se ajustaba a las características académicas, personales y sociales enunciadas con anterioridad.

En líneas generales, se puede concluir que la satisfacción es bastante buena debido a los resultados alcanzados en todos los ítems (medias entre 3.52 y 3.71). Si bien, este valor es superior cuando se le pregunta sobre la tutoría y el tutor en general, que en cada uno de los aspectos particulares, por lo que la muestra encuestada tiene una buena opinión sobre la totalidad del proceso de tutorización y todos los factores que en él intervienen $(\bar{x}=3.86)$. 
La tutoría durante el proceso de desarrollo del TFG y TFM: análisis del grado de utilidad y satisfacción del alumnado

Nuria Rebollo Quintela y Eva María Espiñeira Bellón

Tabla 3. Satisfacción respecto a la tutoría.

\begin{tabular}{|c|c|c|c|c|c|}
\hline & $\mathrm{N}$ & $\bar{x}$ & $\mathrm{Md}$ & Mo & SD \\
\hline \multicolumn{6}{|l|}{ Estoy satisfecho con ... } \\
\hline $\begin{array}{l}\text { Los conocimientos que tenía mi tutor/a sobre la } \\
\text { estructura a seguir con respecto al TFG/TFM }\end{array}$ & 73 & 3.58 & 4 & 5 & 1.443 \\
\hline $\begin{array}{l}\text { Los conocimientos que tenía mi tutor/a sobre la } \\
\text { organización del proceso de tutoría del TFG/TFM }\end{array}$ & 73 & 3.58 & 4 & 5 & 1.290 \\
\hline $\begin{array}{l}\text { El clima de confianza que ha logrado crear mi } \\
\text { tutor/a durante todo el proceso }\end{array}$ & 73 & 3.66 & 4 & 5 & 1.511 \\
\hline $\begin{array}{l}\text { La disponibilidad de mi tutor/a durante todo el } \\
\text { proceso }\end{array}$ & 73 & 3.62 & 4 & 5 & 1.468 \\
\hline $\begin{array}{l}\text { La respuesta que ha dado mi tutor/a a mis necesi- } \\
\text { dades durante todo el proceso }\end{array}$ & 73 & 3.58 & 4 & 5 & 1.423 \\
\hline $\begin{array}{l}\text { El número de contactos que he tenido con mi } \\
\text { tutor/a durante el proceso }\end{array}$ & 73 & 3.71 & 4 & 5 & 1.317 \\
\hline $\begin{array}{l}\text { El nivel de empatía que ha mostrado mi tutor/a } \\
\text { durante el proceso }\end{array}$ & 73 & 3.66 & 4 & 5 & 1.407 \\
\hline $\begin{array}{l}\text { El nivel de agilidad que ha mostrado mi tutor/a } \\
\text { durante el proceso }\end{array}$ & 73 & 3.52 & 4 & 5 & 1.375 \\
\hline $\begin{array}{l}\text { En general, estoy satisfecho con la tutoría del } \\
\text { TFG/TFM }\end{array}$ & 73 & 3.86 & 4 & 5 & 1.388 \\
\hline $\begin{array}{l}\text { En general, el tutor/a ha satisfecho mis expectati- } \\
\text { vas }\end{array}$ & 73 & 3.86 & 4 & 5 & 1.388 \\
\hline
\end{tabular}

\section{Conclusiones}

La experiencia acumulada en estos años de andadura del EEES y con la trayectoria experimentada por los trabajos de fin de grado y máster en las diferentes universidades no sólo nacionales sino europeas, hace que nos encontremos en un momento propicio para repensar. No sólo por la dificultad que entraña la novedad de tal hecho en sí misma, sino que es necesario reflexionar si los cambios realizados a nivel curricular se han acompañado de otros, necesarios, e imprescindibles.

No podemos olvidar, que el proceso de elaboración y defensa del trabajo de fin de grado o máster, no se da de manera solitaria, sino que tiene que ser acompañado en todo momento por la figura del profesorado tutor/a, al que se le atribuyen unas funciones, características, cualidades, ... dependientes de la Universidad o centro, ya que estas no vienen recogidas en el RD 1393/2007, y en muchas ocasiones están condicio- 
nadas por las concepciones sobre la orientación y la tutoría, ligadas al perfil del tutor, siendo como señala Zabalza (2007, p. 141) "aquellos aspectos actitudinales difusos, que se pueden resumir en la forma de ser".

Los resultados obtenidos permiten: conocer y describir los perfiles del estudiantado durante su periodo formativo, analizar la percepción que tiene sobre la utilidad de la tutoría, considerándola en líneas generales necesaria y útil (tanto la de tipo individual como la grupal). Por último, nos ha permitido determinar su nivel de satisfacción con la tutoría recibida y afirmar que este es bastante elevado tanto en cada uno de los aspectos que se contemplan en el instrumento como en la satisfacción global con el proceso. Si bien la muestra ha sido "apropiada para detectar algunos problemas que pueden llegar a contradecir la propia finalidad del TFG" (Garrote de Marcos, 2015, p.13) no es suficiente para generalizar los resultados y efectuar un correcto diagnóstico.

En consecuencia, "aún quedan aspectos pendientes que tratar, que constituyen retos ineludibles a nuestra labor docente y de organización, como es el caso de la variabilidad en la líneas de trabajo ofertadas, el alcance de los TFG, y/o los criterios empleados en la evaluación" (Martínez-Fuentes y Pastor Seller, 2014, p. 90), ahondando a su vez en las características presentes en el profesorado tutor/a, las demandadas y el grado de discrepancia entre unas y otras. Permitiéndonos definir un perfil de profesorado tutor/a acorde a la nueva realidad del espacio europeo de educación superior, sumándonos a los trabajos realizados por Koster, Brekelmans, Korthagen y Wubbels (2005) y Merino Tejedor (2014).

\section{Limitaciones del estudio}

El acceso a la población y a la muestra de egresados/as de la Facultad ha sido la limitación principal del estudio, ya que el alumnado que defiende su TFG/TFM deja de ser estudiante de la Facultad y no existe ningún protocolo establecido para poder establecer contacto directo con él. Se ha tratado, no obstante, de mantener rigor tanto en la recogida de información como en el posterior análisis y discusión de los resultados, conociendo una realidad particular (Stake, 2010), sin intención de establecer leyes universales ni de generalizar los resultados. 
La tutoría durante el proceso de desarrollo del TFG y TFM: análisis del grado de utilidad y satisfacción del alumnado

Nuria Rebollo Quintela y Eva María Espiñeira Bellón

\section{Referencias bibliográficas}

Álvarez, M., y Pascual, M.M. (2012). Propuesta de evaluación del trabajo de fin de grado en derecho. Aula Abierta, 1(40), 85-102. Recuperado de http://digibuo.uniovi.es/dspace/bitstream/10651/21155/1/AulaAbierta.2012.40.1.85-102.pdf

Amor Almedina, M.I. (2016). Evaluación de la Orientación y la Tutoría en la Facultad de Ciencias de la Educación de la Universidad de Córdoba. Educatio Siglo XXI, 34 (1) 93-112. http://dx.doi.org/10.6018/j/253231

Buendía, L., Colás, P., y Hernández, F. (1998). Métodos de investigación en Psicopedagogía. Madrid: McGraw-Hill.

Caicedo Camacho, N. (2015). Trabajos de fin de grado: modalidades, objetivos y competencias a validar. La experiencia de la facultad de derecho de la universidad de Barcelona. Docencia y Derecho, Revista para la Docencia Jurídica Universitaria, 9, 1-11. Recuperado de https://www.uco.es/docencia_derecho/index.php/reduca/article/ view/90

Corbetta, P. (2007). Metodología y Técnicas de investigación social. Madrid: McGraw Hill.

Cremades Andreu, R., García Gil, D., Ramírez Rico, E., y Miraflores Gómez, E. (2016). Acción tutorial en estudiantes de las menciones de educación física y música del grado de maestro en educación primaria. Revista de Investigación Educativa, 34(2), 417-433. DOI: http://dx.doi.org/10.6018/rie.34.2.237931

Díez Bueso, L. (2015). Las responsabilidades del profesor en la dirección de los trabajos de fin de grado. Docencia y Derecho, Revista para la Docencia Jurídica Universitaria, 9, 1-19. Recuperado de https://www.uco.es/docencia_derecho/index.php/reduca/article/ view/93/115

Fernández Riveira, R.M. (2015). La implicación del docente en el trabajo fin de grado: responsabilidades y reconocimiento académico. Docencia y Derecho, Revista para la Docencia Jurídica Universitaria, 9, 1-13. Recuperado de https://www.uco.es/docencia/grupos/reduca/index.php/reduca/article/view/94

Fox, D.J. (1981). El proceso de investigación en educación. Pamplona: EUNSA.

Garrote de Marcos, M. (2015). El TFG: sus modalidades, objetivos y competencias a validar. Reflexiones a partir de la experiencia de la facultad de derecho de la UCM. Docencia y Derecho, Revista para la Docencia Jurídica Universitaria, 9, 1-14. Recuperado de http://helvia.uco.es/xmlui/handle/10396/13626

Gil Pascual, J.A. (2011). Técnicas e instrumentos para la recogida de la información. Madrid:UNED.

Hernández-Leo, D., Moreno Oliver, V., Camps, I., Clarisó, R., Martínez-Monés, A., Marco-Galindo, M.J., y Melero, J. (2013). Implementación de buenas prácticas en los trabajos fin de grado. Revista de Docencia Universitaria, Número especial (11), 259-278. Recuperado de http://polipapers.upv.es/ index.php/REDU/article/view/5556/5547

Koster, B., Brekelmans, M., Korthagen, F., y Wubbels, T. (2005). Quality requirements for teacher educators. Teaching and Teacher Education, 21, 157-176.

López Martín, I., González Villanueva, P., y Velasco Quitana, P.J. (2013). Ser y ejercer de tutor en la universidad. Revista de docencia Universitaria, 11 (2), 107-134. Recuperado de http://polipapers.upv.es/index.php/REDU/article/view/ 5569/0 
La tutoría durante el proceso de desarrollo del TFG y TFM: análisis del grado de utilidad y satisfacción del alumnado

Nuria Rebollo Quintela y Eva María Espiñeira Bellón

Martínez Clares, P., Pérez Cusó, J., y Martínez Juárez, M. (2014). Una (re)visión de la tutoría universitaria: la percepción de estudiantes y tutores de estudios de Grado. Revista de Docencia Universitaria, 1(12), 269-305. Recuperado de https://dialnet.unirioja.es/descarga/articulo/4692022.pdf

Martínez-Fuentes, M.T., y Pastor Seller, E. (2014). El trabajo fin de grado en la facultad de trabajo social de la universidad de Murcia. Revista Internacional de Trabajo Social y Bienestar, 3, 83-91. Recuperado de http://revistas.um.es/azarbe/ article/download/198401/161671

Merino Tejedor, E. (2014). El tutor del trabajo fin de grado: Nuevos desafíos para el profesor de universidad. International Journal of Developmental and Educational Psychology, 1(4), 239-244. Recuperado de http://www.infad.eu/RevistalNFAD/OJS/ index.php/ IJODAEP/article/view/608/546

Merino Tejedor, E., Pinedo González, R., y Gómez Barreto, I.M. (2014). Aportaciones de la psicología humanista a la función del tutor del trabajo fin de grado. Revista de Análisis Transaccional y Psicología Humanista, 71, 211-227. Recuperado de http:// com.aespat.es/Revista/Revista_ATyPH_71.pdf

Ortiz-Repiso, V., García Zorita, C., Pacios, A.R., y Vianello, M. (2014). Planificación y evaluación del trabajo de fin de grado: el caso del grado en información y documentación de la universidad Carlos III de Madrid. Revista General de Información y Documentación, 24(2), 279-304. Recuperado de http://dx.doi.org/10.5209/rev_ RGID.2014.v24.n2.47237

Pérez Cusó, F.J. (2013). Tutoría universitaria: ¿Un elemento de calidad? Un estudio en la Facultad de Educación de la Universidad de Murcia (Tesis doctoral). Recuperado de: http://www.tdx.cat/bitstream/handle/10803/123972/TFJPC.pdf? sequence=1

Pulido Rodríguez, R., Ballén Ariza, M., y Zúñiga López, F.S. (2007). Abordaje hermenéutico de la investigación cualitativa. Teorías, procesos, técnicas. Colombia: Universidad Cooperativa de Colombia.

Rekalde Rodríguez, I. (2011). ¿Cómo afrontar el trabajo de fin de grado? Un problema o una oportunidad para culminar con el desarrollo de las competencias. Revista Complutense de Educación, 22(2), 179-193. Recuperado de http://revistas.ucm.es/index. php/RCED/article/viewFile/38488/37226

Roiss, S. (2015). El trabajo de fin de grado en el grado de traducción e interpretación: evaluación de competencias y contenidos asociados al título. Estudio analítico y consecuencias didácticas. Quaderns, Revista de Traducció, 22, 273-288. Recuperado de http://hdl.handle.net/10366/126618

Stake, R.E. (2010). Qualitative research: studing how things work. New York: The Gilford Press.

Tarí Guilló, J.J, De Juana Espinosa, S., Valdés Conca, J., Andreu Guerrero, R., Manresa Marhuenda, E., Sabater Sempere, V. ... Fernández Sánchez, J.A. (2015). El proceso de elaboración y tutorización de los TFG y TFM a examen. En J. D. Álvarez Teruel, M.T. Tortosa Ybañez y N. Pellín Buades (coord.) Investigación y propuestas innovadores de redes para la mejora de la docente (pp.1358-1375). Recuperado de http://rua.ua.es/ dspace/handle/10045/50789

Todd, M., Bannister, P., y Clegg, S. (2004). Independent inquiry and the undergraduate 
La tutoría durante el proceso de desarrollo del TFG y TFM: análisis del grado de utilidad y satisfacción del alumnado

Nuria Rebollo Quintela y Eva María Espiñeira Bellón

dissertation: perceptions and experiences of final year social science students. Assessment \& Evaluation in Higher Education, 29(3), 335-355 http://dx.doi.org/10.108 0/0260293042000188285

Zabalza, M.A. (2007). Competencias docentes del profesorado universitario. Calidad y desarrollo profesional. Madrid: Narcea, S.A. de Ediciones.

Zumaquero Gil, L. (2015). La acción tutorial en los trabajos de fin de grado: análisis de su funcionamiento en la titulación de grado de derecho de la universidad de Málaga. Docencia y Derecho, Revista para la Docencia Jurídica Universitaria, 9, 1-14. Recuperado de https://www.uco.es/docencia_derecho/index.php/reduca/article/view/9 7 